\title{
VERANTWORTUNG VON UNTERNEHMEN IM UMWELTSCHUTZRECHT
}

\section{Corporate responsibility in environmental protection law}

\author{
CSILLA CSÁK*
}

\begin{abstract}
We can talk about environmental responsibility in several dimensions. It can be seen that legal liability is only part of the question of responsibility. Legal liability, together with the rules set out in the general codes and sui generis provisions of environmental law, also represents a complex system of administrative, civil and criminal liability. Every company reacts differently to environmental responsibility, so we can ask the question: can we talk about corporate responsibility or irresponsibility?
\end{abstract}

Keywords: environment protection law, corporate responsibility, pollution, legal liability

Wir können über Verantwortung im Zusammenhang mit dem Umweltschutz in mehreren Dimensionen sprechen. Die ganze Menschheit ist verantwortlich für die Zerstörung der Schätze der Erde, die gegenwärtige Generation ist verantwortlich für die Erhaltung einer gesunden Umwelt für zukünftige Generationen. Der Staat und alle Personen sind für den Schutz der Umwelt, die Verschmutzung der Umwelt aus moralischen, politischen, religiösen und ähnlichen Gründe verantwortlich. Es ist ersichtlich, dass die rechtliche Haftung nur ein Teil der Frage der Verantwortung ist. Die rechtliche Haftung stellt zusammen mit den in den allgemeinen Kodexen und den sui generis-Vorschriften des Umweltrechts festgelegten Regeln auch ein komplexes System der administrativen, zivil- und strafrechtlichen Haftung dar.

Hinter der rechtlichen Verantwortung steht der organisierende Zwangsapparat des Staates. Die Regelung der gesetzlichen Haftung muss sehr klar, abgegrenzt, kohärent und anwendbar sein. Nicht nur die Gesetzgebung, sondern auch die Rechtsanwendung müssen diese Bedingungen erfüllen, um eine einheitliche Auslegung des Gesetzes zu erreichen.

Neben dem Rahmen des nationalen Rechtssystems taucht das Thema der Haftung auch in internationalen und EU-Bereichen auf, die den Inhalt der Umweltbeziehungen durch „weiche“ und „harte“ Regulierungsbehörden beeinflussen und bestimmen.

Die Bereiche des Privatrechts und des öffentlichen Rechts müssen bei der Regulierung voneinander getrennt betrachtet werden, weil die Regulierungsmethoden

* Dekanin, Universität Miskolc, Staats- und Rechtswissenschaftliche Fakultät. 
aufgrund der Natur dieser beiden Bereiche unterschiedlich sind. Allerdings verwischt im Allgemeinen die scharfe Grenze zwischen öffentlichem und privatem Recht. Die Grenzen und die Haftungsbereiche bestimmen den Rahmen des menschlichen Verhaltens und den Standard unter dem das menschliche Verhalten beurteilt und in einigen Fällen unterschiedlich bestraft wird.

Im Bereich der umweltrechtlichen Haftung ist die verwaltungsrechtliche Regelung vorherrschend, was auf das Übergewicht des staatlichen Einflusses hinweist. Gleichzeitig spielen aber auch Wirtschaftsregulierungsinstrumente, freiwillige Instrumente und Konsensinstrumente, die den Einfluss des Staates weniger oder indirekt widerspiegeln, eine immer wichtigere Rolle. Durch diese Instrumente erfordert die Gesetzgebung die vollständige Vertikale von Instrumenten des öffentlichen und privaten Rechts. Unter den zivilrechtlichen Rechtsinstitutionen sind auch der rechtliche Schutz von Personen, Sachenrechte, das Recht der Schuldverhältnisse und die Theorien der Verantwortung in das komplexe System der umweltrechtlichen Regulierung integriert. In Bezug auf das Strafrecht kann eine Reihe von Tatsachen hervorgehoben werden, insbesondere Umweltschädigung, Beeinträchtigung der Natur und Verletzung der Ordnung der Abfallbewirtschaftung.

Die Frage ist, was der Zweck der Regelung ist und mit welchen Mitteln sie erreicht werden kann?

Ziel der Regelung ist der Umweltschutz in einem besonders hohen Umfang, die Vermeidung der Umweltverschmutzung und den Umweltschäden, die Beseitigung der verursachten Schäden und die Wiederherstellung des ursprünglichen Zustands. Das zentrale Element ist die Prävention. Viele der Präventionsinstrumente können mit Vorschriften im Verwaltungsbereich verknüpft werden, insbesondere mit Genehmigungsverfahren. Die Rolle anderer Rechtsbereiche hängt stärker mit der Reparatur zusammen.

Neben dem Umweltinteresse existiert jedoch auch ein starkes wirtschaftliches Interesse. Umwelt- und Wirtschaftsinteressen konkurrieren miteinander. Dies zeigt sich auch in den Entscheidungen der Rechtsanwendungsbehörden. Wenn man sich die Rechtsprechung anschaut ist festzustellen, dass die Gerichte die Rechtsfolge eines Verbots, einer Aussetzung oder einer Einschränkung der Tätigkeit sehr selten anwenden. Der Grund dafür kann in vielen Fällen auf wirtschaftliche Probleme (Beschäftigung, soziale Situation, Investitionen usw.) zurückgeführt werden. Daher bleibt die Geldbuße oder die Wiedergutmachung, das heißt die finanzielle Entschädigung, meistens bestehen.

Deswegen sind die Aspekte der Schadensbehebung und die Verbesserung der Leistungsfähigkeit von größter Bedeutung. Das finanzielle Sicherheitensystem, z. B. die obligatorische Haftpflichtversicherung funktioniert in Ungarn nicht auf Systemebene. Dazu gehört aber auch die Errichtung verschiedener Umweltfonds. Auf Unionsebene gibt es verschiedene Solidaritätsfonds im Falle von Umweltkatastrophen. Wir können zwischen zwei Arten von Umweltkatastrophen unterscheiden. Es gibt einen Ausgleichsfonds für Naturkatastrophen, aber keinen für Industriekatastrophen. Die Katastrophen beschleunigen im Bereich des Umweltschutzes erheblich die Ge- 
setzgebung auf EU-Ebene. Die Rotschlammkatastrophe, die auf die Mängel der Regulierung von Finanzsicherheiten nicht nur in Ungarn, aber auch auf EU-Ebene hinwies, veranlasste die Europäische Kommission, im Falle von Industriekatastrophen mit der Einrichtung des Finanzfonds zu beginnen.

In Bezug auf das Sicherungssystem besteht die Grundvoraussetzung darin, dass einerseits den Wirtschaftsteilnehmern Finanzdienstleistungen zur Verfügung stehen und zu vertretbaren Kosten gehalten werden können, und andererseits, dass sie die finanzielle Belastung durch die Sanierung effektiv abdecken. Leider kann nach derzeitiger Erfahrung kein finanzielles Sicherungsinstrument für die Umsetzung eines komplexen Zielsystems geeignet sein. Die Art und Weise, wie die Werkzeuge verwendet werden, hängt auch von den Parametern der jeweiligen Wirtschaftsorganisation und der von ihr durchgeführten Umweltnutzungsaktivität ab. ${ }^{1}$

Das System, Gewicht und die Art der Sanktionen sollten also nicht nur bei der Wiedergutmachung, sondern auch bei der Prävention eine wirksame Rolle spielen sollen und können. Die bei der Feststellung der Haftung anzuwendenden Sanktionen (typischerweise Schadensersatz) dienen nicht primär der Schadensabwehr und der Wiederherstellung des ursprünglichen Zustands. Es muss ein komplexes System von Entschädigungsmodellen implementiert werden, das eine Lösung sucht, bei der sowohl die geschädigte Umwelt als auch die durch die illegalen Umweltschäden Geschädigten entschädigt werden. Sie sucht auch nach einer Lösung, bei der der Schädiger über angemessene Garantien verfügt, um die nachteiligen Folgen des rechtswidrigen Verhaltens zu beseitigen. Im Bereich der gesetzlichen Regulierung ist das Sicherheitensystem das schwächste Kettenglied des komplexen Modells. Die Regulierung des Sicherheitensystems und die Einrichtung eines einheitlichen Systems sind sowohl auf EU- als auch auf nationaler Ebene von wesentlicher Bedeutung. Die konzeptionelle Möglichkeit der Einrichtung eines verpflichtenden Sicherungssystems in der EU wird sich abzeichnen. Es ist eine bedauerliche Tatsache, dass die effektive Entwicklung einiger Elemente des Systems im Allgemeinen nach einer schweren Umwelttragödie stattgefunden hat. Zu den Zielen der Haftung gehört es, eine finanzielle Grundlage für die Sanierung der Umwelt zu schaffen. Die Rehabilitation selbst erfolgt aus einer Reihe von Zwecken (sozial, ökologisch, wirtschaftlich), aber die Haftung setzt auch voraus, dass sie nicht unbedingt kollektiv abgedeckt werden muss.

Das ungarische Grundgesetz enthält sehr eindringlich den Schutz von Umweltwerten und Interessen, der an mehreren Orten im Gesetz durch detaillierte Regulierungselemente dargestellt wird; beispielsweise das Recht auf eine gesunde Umwelt, der Schutz der natürlichen Ressourcen, der Schutz der Rechte künftiger Generatio-

1 Im Kontext anderer Umweltgesetzgebungen, wie der Abfallwirtschaft, wurden viele Erfahrungen mit Produkten außerhalb des Versicherungsmarktes (Bürgschaft, Bankgarantie, Fonds, Einsatz eines „eigenen“ Versicherers usw.) gesammelt. Diese Instrumente können in der Regel mit geringen Änderungen an die Haftung im Geltungsbereich der Richtlinie angepasst werden. 
nen, der gentechnikfreien Landwirtschaft, die Regulierung der Abfallbewirtschaftung und die Anhebung des Verursacherprinzips auf das verfassungsrechtliche Niveau. Generell kann festgestellt werden, dass das ungarische Grundgesetz auch auf Ebene der Mitgliedstaaten eine starke Umweltorientierung aufweist.

Das wirtschaftliche Interesse zeigt sich auch auf der Seite der Umweltnutzer. Aufgrund mangelnden Umweltbewusstseins kann ein klima- und umweltfreundliches Verhalten effektiv mit wirtschaftlichen Mitteln erreicht werden. Solche wirtschaftlichen Instrumente sind, z. B. Steuern, Nutzungsgebühren oder Subventionen. Gleichzeitig existieren die freiwilligen Umweltinstrumente, die sich für das Umweltbewusstsein von Unternehmen einsetzen, z. B. Prüfungssysteme (ISO, EMAS) oder das Umweltabzeichen.

Die Unternehmensleiter haben auch eine Verantwortung gegenüber Eigentümern, Mitarbeitern und der Gesellschaft. Natürlich erscheinen die Art und der Umfang der Verantwortung in verschiedenen Branchen völlig unterschiedlich und implizieren moralische und rechtliche Verantwortung für finanzielle Stabilität, existenzielle Sicherheit, Beschäftigung und die Beseitigung nachteiliger sozialer Auswirkungen. Dies schließt die soziale Reaktion auf Umweltkatastrophen ein, die ein intensiveres Zur-Verantwortung-Ziehen erfordert. Dieser Prozess hat die Anwendung einer zunehmenden zivil- und strafrechtlichen Haftung in Westeuropa erzwungen. Infolge des angewandten Sanktionssystems kann ein Unternehmen nicht nur durch eine schlechte finanzielle Entscheidung, sondern auch durch ein Umweltmanagement auf der Grundlage falscher Mechanismen zerstört werden, was negative Auswirkungen auf sozialer Ebene mitsichführt.

Die Auswirkungen von Umweltherausforderungen auf wirtschaftliche Prozesse sind zweifellos nicht unerheblich. Die Folgen der steigenden Kosten werden auch von Wissenschaftlern erkannt und unterstützt. In Kanada und den USA gibt es eine zunehmende Anzahl von Fällen, in denen die Manager auch für die umweltschädlichen Aktivitäten eines Unternehmens strafrechtlich haften können. In der Gesellschaft wächst die Forderung, diejenigen zu bestrafen, die umweltschädliche Tätigkeiten ausüben. Die Behörden reagieren auf diese soziale Forderung, wodurch in vielen Fällen sehr harte Strafen verhängt wurden.

Die Unternehmensleiter müssen vor allem die gebotene Sorgfalt walten lassen und müssen Umweltprüfungssysteme auf Ebene des Umweltmanagements und Audit entwickeln und betreiben, die das Ausmaß der Umweltrisiken minimieren und somit die Einhaltung der Umweltvorschriften sicherstellen. In Ungarn ermöglichen spezielle gesamtschuldnerische Haftungsregeln eine Erweiterung des Kreises der Verpflichteten.

Eine Schadensersatzpflicht als Lösung, auch wenn sie in einem langwierigen und vor allem kostspieligen Rechtsstreit entsteht, ist nur bedingt befriedigend. Das verschmutzende Verhalten dauert in der Regel länger an, der Täter fühlt sich oft zu Unrecht bestraft (Umkehr der Verantwortung), das Opfer wird durch das Geld getröstet, aber nicht beruhigt, weil der Schaden länger anhält oder weil eine Sanierung der belasteten Umwelt nicht möglich ist. Eine Haftpflicht, also die nachträgliche Be- 
seitigung von Umweltschäden, eine durch bilateral erzwungene Kompromisse belastete Lösung, ist nicht die beste Lösung, da sie zwar eine subjektive Sanktion ist, aber der Funktion der Prävention und Bildung für die Zukunft kaum gerecht wird. Sie ist zudem „unsicher“ für die Schädigerseite und deckt den Reparaturbedarf nur bedingt oder ist dazu gar nicht in der Lage. ${ }^{2}$

Die einschlägige Rechtsprechung umfasst jedoch fast ausschließlich Schadensersatzfälle, eine Haftung ist auch dann begründet, wenn die Klage unter einem anderen Titel eingereicht wurde (Hausfriedensbruch, Nachbarschaftsanspruch). Es scheint, als sei dies aus mehreren Gründen die „billigste“ Lösung, weil die Entschädigungshöhe immer geringer ist als der Preis der Kläranlage oder gar der Verlust durch die Stilllegung der Anlage. Dies gilt auch im übertragenen Sinne, denn es besteht keine Notwendigkeit, das Zivilrecht mit seinen spezifischen Anpassungsprobleme an die immer komplexeren gesellschaftlichen Verhältnisse zu verschärfen oder weitergehende Zusammenhänge einzubeziehen, die zu weit in die Sphären der Wirtschaftspolitik, der wirtschaftlichen Ebene oder der administrativ-organisatorischen Sphären reichen. Zugleich ist diese Bipolarität des Rechtsverhältnisses fragwürdig, da die neue Dimension des Umweltrechts die Berücksichtigung gesellschaftlicher Interessen erfordert.

Auch aus wirtschaftlicher Hinsicht lässt sich die Frage eindeutig beantworten und wir können zu einem ähnlichen Ergebnis kommen wie bei der gesetzlichen Regelung, nach dem juristischen Ansatz, aber auch auf der Grundlage des gesunden Menschenverstandes. Bei diffusen Externalitäten bestimmter negativer Natur ist die zentrale Frage, ob die externen Auswirkungen (Schäden) auf eine Produkteinheit (Dienstleistung) die positiven Auswirkungen ${ }^{3}$ der beabsichtigten Tätigkeit übersteigen. In diesem Fall muss die Gemeinde (der Staat) das betreffende Unternehmen oder die betreffende Tätigkeit liquidieren, auch wenn es ansonsten rein marktorientiert profitabel ist. Wenn der Nutzen des Kerngeschäfts die negativen externen Effekte überwiegt, sollte das Unternehmen dann die Opfer oder die Gesellschaft im Verhältnis zum verursachten Schaden entschädigen? ${ }^{4}$ Hinsichtlich des Zeithorizonts der Haftung sollte die Vorbeugung und Beseitigung schädlicher Auswirkungen als vorrangiges Ziel und Verpflichtung betrachtet werden. Die Beseitigung der Benachteiligungsfolgen, die Feststellung der Haftung des Betroffenen, die Durchsetzung der

2 LENKOVICS Barnabás: A polgári jog lehetöségei a környezetvédelemben. http://www. kvvm.hu/szakmai/karmentes/egyéb/kjogalk/02.htm (25. Januar 2012).

3 Grundsätzlich kann es natürlich auch sein, dass die positive Außenwirkung eines Unternehmens oder einer Tätigkeit auf eine Produkteinheit (Dienstleistung) den Nutzen aus dem Kerngeschäft übersteigt. Mag es sogar sein, dass das Kerngeschäft des Unternehmens unrentabel ist, seine positive Außenwirkung aber im Allgemeinen günstig ist. In diesem Fall muss die Gemeinschaft (der Staat) die Tätigkeit unterstützen, auch wenn sie ansonsten rein marktverlustig ist - triviale Beispiele hierfür sind Landesverteidigung, Strafverfolgung, Bildung, Heilung usw. TÓTH I. János: Az externália új dimenziói. Magyar Tudomány, 2008/05, 593.

4 TótH: a.a.O. 593. 
Haftung und die wirksame Durchsetzung der Rechtsfolgen sind ebenfalls ernstzunehmende Herausforderungen und wesentliche Aufgaben für die öffentliche und private Strafverfolgung und die Gesellschaft. Ein wirksames Instrument hierfür ist die Frage der konsequenten Durchsetzung der Privathaftung. Alle Elemente der Haftung müssen jedoch geregelt, anwendbar und durchsetzbar sein. Leider weisen Umweltkatastrophen in vielen Fällen auf Mängel bei der Strafverfolgung hin, wie beispielsweise die Rotschlamm-Katastrophe auf das unzureichende Funktionieren des Finanzsicherheitssystems. Die finanziellen Auswirkungen bestimmter Umweltkatastrophen können die finanzielle Tragfähigkeit des Umweltnutzers, gegebenenfalls aber auch des betreffenden Staates, überwiegen.

Mit den Worten von Gyula Eörsi wird „die Verantwortung im sozialen Sinne durch ein für eine beschuldigte Gesellschaft gefährliches Verhalten ausgelöst. Verantwortung ist der Teil der gesellschaftlichen Bildungsfunktion, der auf gesellschaftlich gefährliches Verhalten mit Repression reagiert." ${ }^{5}$ Umweltverantwortung im weiteren und engeren Sinne, nach dem Verursacherprinzip und in seiner Verfassungserklärung, ist ein Instrument für eine nachhaltige Entwicklung, zum Schutz der Interessen künftiger Generationen.

Jedes Unternehmen reagiert unterschiedlich auf Umweltverantwortung, sodass wir die Frage stellen können: Können wir über die Verantwortung oder die Unverantwortlichkeit der Unternehmen sprechen?

\section{LITERATURVERZEICHNIS}

[1] LENKOVICS Barnabás: A polgári jog lehetöségei a környezetvédelemben. http:// www.kvvm.hu/szakmai/karmentes/egyéb/kjogalk/02.htm (25. Januar 2012).

[2] Tóth I. János: Az externália új dimenziói. Magyar Tudomány, 2008/05.

[3] EÖRSI, Gyula: Kötelmi jog. Általános rész. Nemzeti Tankönyvkiadó, Budapest, 2003.

5 EÖRSI Gyula: Kötelmi jog. Általános rész. Nemzeti Tankönyvkiadó, Budapest, 2003, 239. 